\title{
Research on the Application of PDCA Theory in Nursing Quality Management
}

\author{
Yanxia Wang ${ }^{1, a}$, Shiyu Zheng ${ }^{1, b}$ \\ ${ }^{1}$ Affiliated hospital of Beihua University, Jilin 132011, China. \\ ayanxia_wang@163.com, bshiyu_zheng@126.com
}

Keywords: PDCA theory, Nursing quality, Nursing management.

\begin{abstract}
This study put the theory of PDCA (Plan, Do, Check, Action into ) the quality of nursing management; research the role of PDCA in the operation of nursing skills, the first-aid inventory management, the basic nursing knowledge and the patients' attitude towards nursing service. It helps to explore a road of development in nursing career which fits Jilin region.
\end{abstract}

\section{Introduction}

Since the founding of hospital quality of care experienced by the qualitative management to quantitative management, management experience to the development of scientific management. 30 years after the founding of new China among nursing management experience mainly in the management of the main, or "paternalistic" or "Butler" management methods, the lack of reliable scientific, so difficult to guarantee the quality of care. Since the 1990s, with the exchange of experience and cooperation with domestic and foreign nursing career, nursing management into our overall health reform, and actively explore with the international standards of care management model on the road, we began a systematic holistic nursing pilot promotion. Holistic Nursing (Systematic Approach to Holistic Nursing Care, Acronym SHN) is a patient-centered, modern nursing concept as a guide to nursing process as the core, care management and clinical care of all aspects of the business combination in accordance with the nursing process scientific methods of work, for clients to provide solutions to the problem of health, the implementation of effective overall care. As we all know, WHO's "Health is not only no physical illness, but also a complete physical, mental state and a good social adaptation" has been replaced by a defined people's attitudes about "Health is no disease" of the ages; realization by the biomedical model to the physical, psychological, social change medical model. At the same time, the development of nursing work also achieved by the "disease-centered" care system function to the "patient-centered" care of the whole followed again "to human health as the center" holistic nursing model difficult transition. So now we care services including not only the patient, but also healthy people, from the traditional single nursing care management model to diversify management.

The current outstanding problems facing the management of hospital care generally has four: First, the needs of patients undergone new changes. Not only to meet the general health care needs, but also to provide a higher quality of health care services, the only way to meet the growing demand for health care services; the second is the overall care needs to be deepened further reflected in the patient-centered; the third is health care quality management and improve the fish to be standardized: four is gradually replaced by public health insurance, patients need low medical expenses. Who "Let the patient a speedy recovery, reduce medical costs, who will win the trust of patients, grab opportunities in the medical market." With the deepening of the overall care, the new care model Meaning new meaning to nursing management, nursing management faces cross-century concept of change and strategic choices, calling for new thinking, new ideas, new strategy and new methods of nursing management . At present, China is still in a state of nursing management experience in management. Nursing management decisions depend largely on past experience, and the lack of more specific and precise data support and theoretical guidance. Based on the extensive literature in-depth study, through empirical research in Jilin City, some hospitals, the PDCA management theory and experience traditional management methods were compared to explore the region for the present 
stage of development of the nursing profession road. More effective, more humane, more scientific management methods applied to the quality of care in nursing quality management to continuously improve the quality of care, improve patient satisfaction, improve medical quality, reduce nurse-patient conflicts.

\section{Intervention}

In a hospital in the experimental group rose hospitals as an opportunity for hospital nurses batches at mobilization training, to promote the PDCA theoretical knowledge in nursing staff in the hospital, people who can master and skilled use of PDCA Theory work in day care, develop good work habits, rigorous style of work, really do write what I do, do what I wrote: organic draw systematically carry out every task, from the self-start , good self and mutual inspection, nursing random checks, periodic inspection department. Formation of full participation, the entire process of managing a good situation. The integration of individual ring in PDCA loop and small departments, snowballed to promote the hospital's large PDCA loop ladder rise again and again.

Four stages of PDCA cycle is not completely separate, but closely co-coordinated fused, there will be a certain amount of cross phenomenon between stages. In practice, often the side edge inspection program implementation side, while checking side edge adjustment plan summary, that is, not mechanically to rotate the PDCA cycle. Specific workflow: Presented Goals - Understanding the problem - to analyze the reasons - to develop measures - implementing measures - check the effect or lessons learned into the next PDCA.

(1) planning phase $\mathrm{P}$ (Plan)

Understanding the problems identified in the quality of care nurses checking operation is not standardized by the examiner pointed out that after documenting nursing department reported. Nursing Care in technical operations organized by the Ministry, the invigilator in scoring while on emerging issues to the parties, and after finishing problems reported to the Department of Nursing. Analyze the reasons nurses operating standards are not fully grasp, sterile concept is not strong, in many patients, the peak of work often appears streamline workflow, legal sense of apathy, in operation, exchange and communication with the patient is not in place, can not fulfill the obligation , the only operation, the operation stiff action, pay attention to observe the patient's response is not strong sense of responsibility to individual nurses. Develop measures for common problems occur by reason it is easy to organize hospital care unit nurses study "Jilin technical operation quality evaluation criteria," individual problems of individual people notice head nurses, head nurses supervise individual rectification. Make it clear operating standards, the head nurse at the peak of the work to implement flexible scheduling, increase caregiver. Legal awareness and enhance the sense of responsibility.

(2) the implementation phase D (Do)

Care Ministry of hospital nursing technical operations training programs, the establishment of the Group of Experts nursing techniques. Send technical backbone of the operation to study in technical operating standards back to the hospital nursing department and members of the Group, the technical backbone of a unified operating standards, and batch-operation in the hospital-wide demonstration. Stressing the operation, operation after operation three key aspects of the process, emphasizing the principles of aseptic technique, etiquette and nurses in nursing techniques in the application. Nursing Department staff manage technical operations, field operations and nursing staff of the department, the group members and technical backbone of the scene to guide the operation until the master, and the new posts pre-service training nurses. Nursing Department hired well-known experts to give lectures, organize the nurses studied law and regulations, nurses etiquette, communication skills and other humanities knowledge, strengthen legal awareness, safety awareness and informed awareness and continuously improve the overall quality of nurses. Stressed that the assessment before the operation, and actively carry out exchanges and communication patient, the patient's psychological understanding, empathy really do for the sake of the patient. For example, when a patient catheterization, enema care operations involving personal privacy, by assessing before the operation, 
so that patients understand the operating precautions, reduce patient fear and anxiety, the operation for patients obscured the real achieve the "patient-centered" humane care, improving quality of care and services.

(3) Check the phase $\mathrm{C}$ (check)

Nursing department random checks once every quarter, all personnel at the scene examination ballot, members of the Group examiner, live scoring and give spot guidance on common problems escalation nursing, unified training by the Department of Nursing, and the next PDCA audits. Individual problems with individual departments who appear nurse contacted by the department head nurse supervision and inspection. Department head nurse once a month for undergraduate nursing assessment, nursing expert group on the issue resolved in a timely assessment, all nurses participate in a unified examination, unified scoring, nurses mutual evaluation process, both found themselves from other people's problems problem, but also to find the gap from someone else's results. For we live discussion of the problems identified, jointly proposed improvement measures, within a time limit, and as the contents of the next PDCA verify. Appraisal process, focus on strengthening humanistic care nurses, with full respect for the patient, emphasizing patient-centered, attention to exchanges and communication with patients, so that patients actually enjoy the right to information, the right to choose, improve patient satisfaction, while improving quality of care.

\section{Hospital nursing quality management process standardization (PDCA) Significance}

PDCA theory in nursing quality management, its advantages: First, the user is not only the patient, but also nurses, nurses recognize that quality of care is a key factor to improve the impact, only the satisfaction of nurses will have the satisfaction of patients. Second, management is service-oriented, emphasizing the role of each nurse. Third, managers no longer control and authority, but the authority given to nurses, support the work of nurses and strengthen exchanges and nurses, guidance and training nurses for nurses to provide services and the talent continue to create the conditions. Fourth, the nurse is no longer a passive work, but to understand the work schedule and plan, clear objectives, the initiative to improve the quality of the owner. Fifth, the quality continues to improve, improve a planned arrangement, there is progress.

Department of Nursing Care Quality Management Committee has, to hospital quality of care quality control, quality control group checks once a month, every quarter time, check the results back to the nursing unit; quality control department nursing staff of the department of the quality of care a week. checks once a month, check, to solve the problem of nursing feedback, success stories submitted nursing care unit at the hospital after normalization by the share; nurses daily self-test; forming a full mobilization, full control, full good situation process management. Meanwhile, the nursing department to hire experts to lecture the hospital, department head nurses supervise nursing rounds and business learning through other forms of learning nurses, nurse encouraged to participate in self-examination, etc., and send outstanding nurses to go out for further study, the hospital carried out a large lecture back to hospital , creating in the hospital-wide positive learning environment; and 5.12 at the end of each year to outstanding nurses reward, the unfinished task of the department or individual criticism. Layers of inspection and supervision to complete the work, study and education to enhance the overall quality of nurses, reward and punishment is an incentive, in all aspects of the application of PDCA nurses from the outside to change the concept, I changed to dry for me to do, consciously take the initiative to complete each the work.

\section{The Impact PDCA quality of nursing techniques}

Studies have shown that, before the intervention group PDCA basic nursing technical operations pass rate of $91.67 \%$, after PDCA intervention group based nursing techniques a pass rate of $100 \%$, after the implementation of PDCA management, basic nursing techniques pass rate has improved significantly. On the basis of quality of care, nursing documentation quality of writing, nursing techniques, special care a quality of care, nurses theoretical level five indicators were observed, 
empirical findings after applying PDCA management, the five indicators showed an increasing trend in hospital quality of care has been continuous improvement. Nursing techniques is an important part of nursing, and patient safety is closely related to the level of care level marks its technological level and the level of quality of care. When nurses nursing technical training, assessment, to emphasize the nurse etiquette. A good image of nurses not only embodies the spirit nurse, returned to the patient to bring comfort and security. Good professional etiquette image only shows the nurse outside the United States, but also deeper, richer content.

\section{Summary}

2 years after the implementation of PDCA management, hospital quality of care has improved steadily over the previous, and an upward trend. Empirical studies have shown that PDCA theory in nursing quality management.

1 nursing techniques, the implementation of PDCA management can improve the rate of qualified nursing techniques;

2. In the first aid items management, the PDCA management, first aid items in good condition to ensure $100 \%$;

3 nurses basic theoretical knowledge, implementing PDCA management, nurses can improve the level of basic theory.

4 patient care satisfaction, implementing PDCA management can improve patient satisfaction.

\section{References}

[1] Wang Hong, Deng Shengping situation and development trend of nursing quality management of People's Liberation Army Hospital Administration Journal, 2000,7 (1): 37-39.

[2] Lidian Fu. Jilin Province booming health. 55th anniversary speech.

[3] Board.N, CaP1an.G Implications of decreasing surgical lengths of stay Australian Health Review; 2000,23 (2):. 62-76.

[4] Hu Yong Yu Hua Shan Fan red, etc. Discussion thinking nursing quality management innovation of Chinese Nursing Management Journal, 2002,18 (9): 565.

[5] Xie Hongzhen On the current challenges facing the nursing management and their future development ideas Chinese Hospital Management, 2001,21 (5): 29.

[6] Wang whole, Zhao Xiaoyun development of nursing quality management of nursing research, \$ 200, 22 (9): 2349.

[7] Lin Juying Hospital Management Nursing Management Volume Beijing: People's Medical Publishing House, 2003.160.

[8] Serious Modern Hospital Medical Quality Management Beijing: People's Medical Publishing House, 2002,165-166.

[9] Progress Ping, Lu Yan, Xu Zhaoyan, etc. .PDCA cycle in the field of nursing applied Journal of Nursing Administration, 2003,20 (4): 47-49.

[10]Zhang Wenli PDCA cycle in nursing management applications: [MS Thesis] Jilin: Jilin University, 2007. 